\title{
Osteoprotegerin prompts cardiomyocyte hypertrophy via FAK/BECLIN1 mediated autophagy inhibition
}

anna shen ( $\sim$ ANNASHEN1@MAIL.COM )

Southern Medical University https://orcid.org/0000-0003-2247-1957

Dezhong Zheng

Southern Medical University

Tingrong Liu

southern medical univeristuy

Tao Zhou

Southern Medical University

\section{Research}

Keywords: Osteoprotegerin, Focal Adhesion Kinase, cardiomyocyte, hypertrophy, autophagy

Posted Date: December 17th, 2019

DOI: https://doi.org/10.21203/rs.2.19019/v1

License: @ (i) This work is licensed under a Creative Commons Attribution 4.0 International License.

Read Full License 


\section{Abstract}

Aim It has been reported that Osteoprotegerin (OPG) induces cardiomyocyte hypertrophy, but the mechanism remains unclear. This study was to investigate the role of Focal Adhesion Kinase (FAK) pathway in the OPG induced hypertrophy in cultured cardiomyocytes.

Methods The H9C2 line of rat cardiomyocytes were treated with OPG at different concentrations and the cellular hypertrophy was evaluated. Meanwhile, the activity of FAK and other the phosphorylation kinases were detected. Autophagy flux assay was performed in absence and presence OPG. The interaction between proteins was analyses using Co-Immunoprecipitation assay.

Results We found that OPG induced cardiomyocyte hypertrophic response, accompanied by dramatic increases a series of inflammatory factors and cytokines, as well as collagen synthesis. Also OPG inhibits autophagy and induces FAK phosphorylation. FAK silencing using si-RNA abrogates the effect of OPG on autophagy and cellular hypertrophy. Furthermore, Co-Immunoprecipitation assay reveals that OPG inhibits autophagy through enhancing the binding of FAK and Beclin1 Tyr 233.

Conclusion The FAK/Beclin1 signal pathway is essential for the OPG induced autophagy inhibition and hypertrophic response in cultured $\mathrm{H} 9 \mathrm{C} 2$ cells.

\section{Background}

As a major complication of hypertension, left ventricular hypertrophy (LVH) is characterized by increased volume and myofibrillar protein content of cardiomyocytes $[9,17]$. LVH leads to thickness of ventricle wall and is regarded as an independent risk factor for cardiovascular morbidity and mortality. To date, the mechanism for LVH has not been well understood.

Osteoprotegerin (OPG) is a member of the tumor necrosis factor receptor superfamily of cytokines and a soluble receptor for the receptor activator for nuclear factor-KB ligand (RANKL) [2, 3]. It has been shown that OPG stimulates cardiomyocyte hypertrophy, indicated by increased cell surface size, protein synthesis per cell, and a series of hypertrophy-related markers [18]. Consistent with this in vitro study, a clinical study showed that OPG in the coronary circulation is associated with concentric LVH [8]. However, the mechanism under which OPG regulates cardiomyocyte hypertrophy warrants further study.

It is well established that the adhesion and accumulation of extracellular matrix (ECM) is necessary for LVH development [17]. The accumulation of ECM is mediated mainly by integrin / Focal adhesion kinase (FAK), which serve as mechanotransducers during normal heart development and in response to physiological and pathophysiological signals such as high blood pressure [20]. As a primary integrin effector, FAK is rapidly activated by mechanical stimuli in cultured neonatal rat ventricular myocytes and in overloaded myocardium of adult animals [15]. In endothelial cells, OPG activates FAK /steroid receptor coactivator ( $\mathrm{Src}$ ) / extracellular signal-regulated kinases (ERKs) signaling and FAK phosphorylation is associated with the morphological changes[12]. A recent study further confirmed that FAK activation 
suppresses autophagy and initiates hypertrophic growth in cultured cardiomyocytes [5]. Based on these findings, we hypothesize that FAK mediated the OPG induced cellular hypertrophic process. In recent years, cardiomyocyte autophagy has been considered to play a role in controlling the hypertrophic response, but the conclusions are controversial. We thus invested the putative role of autophagy in OPG induced cellular hypertrophy in cultured cardiomyocyte.

\section{Methods And Materials}

\section{Cell culture}

Rat cardiomyocyte $\mathrm{H} 9 \mathrm{C} 2$ cells were maintained in DMEM containing $10 \%$ heat-inactivated foetal bovine serum (Life Technologies, Carlsbad, CA, USA) in an incubator in an atmosphere of $5 \% \mathrm{CO}_{2}, 95 \%$ air at $37^{\circ} \mathrm{C}$. After achieving $70-80 \%$ confluence, cells were treated with recombinant human OPG (R\&D Systems ,Mineapolis, MN, USA) at indicated concentrations (see in the results session)

\section{Morphologic assays in Cardiomyocytes}

Cells were grown on the Nunc ${ }^{\mathrm{TM}}$ Lab-Tek $^{\mathrm{TM}}$ II Chamber Slide ${ }^{\mathrm{TM}}$ System (ThermoFisher scientific, USA) to examine changes in cell morphology after OPG treatment. Briefly, cells were gently washed with Phosphate Buffered Saline (PBS) and fixed in 4\%paraformaldehyde, followed by a staining with fluorescein isothiocyanate-conjugated Phalloidin (Sigma-Aldrich, St. Louis, MO, USA) for $30 \mathrm{~min}$. Cellular hypertrophy was evaluated by measuring cardiomyocytes surfaces using a digital image analysis system (Leica QwinV3, Leica Microsystems Ltd, Cambridge, UK). Five random fields (with approximately 10-15 cells per field) from every sample were averaged and expressed as $\mathrm{um}^{2} /$ cell. All experiments were repeated 3 times.

\section{Measurement of Protein Synthesis in Cardiomyocytes}

The $\mathrm{H} 9 \mathrm{C} 2$ cells were gently washed with Phosphate Buffered Saline and trypsinized ( $0.25 \%$ trypsin, Thermo Fisher Scientific, USA ) and counted using a cell counting chamber (Beckman Coulter, Fullerton, $\mathrm{CA}$ ) and then lysed. The cell lysates were prepared to determine protein content by Bradford protein assay. Then the protein synthesis of cells was determined by dividing the total amount of protein by the number of cells, namely, protein per cell.

\section{Western blot assay}

Cultured cells were seeded $\left(1 \times 10^{5} \mathrm{cell} / \mathrm{ml}, 10 \mathrm{ml}\right)$ in cell culture dishes. Concentrations of protein in whole cell extracts were determined using the BCA protein assay kit. FAK, Akt,ERK, PI3K, MAPK and their phosphorylated antibodies were purchased from Santa Cruz Biotechnology (Santa Cruz, CA). Binding of secondary antibodies was detected using anti-rabbit or anti-mouse IgG-HRP $(1: 2,000$, sc-2005, Santa Cruz Biotechnology). Image acquisition was performed on an enhanced chemiluminescence detection system 
(Tanon, Shanghai, China). ImageJ software was used to quantify the density of the specific protein bands.

\section{Mutant plasmid and siRNA transfection}

Beclin1 mutant (Tyr-233 to Phe-233)was generated using QuikChange Site-Directed Mutagenesis Kit (Agilent Technologies,USA). The plasmid was transfected into cells using lipofectamine 3000 (Thermo Fisher Sciencetific) and cells were selected for $72 \mathrm{~h}$ using DMEM supplemented with $2 \mu \mathrm{g} / \mathrm{ml}$ puromycin. The si-RNA sequence against target genes (FAK,ERK,PI3K,MAPK,BECLIN1) and non-targeted (NT) siRNA oligonucleotides were purchased from Dharmacon Research Inc (Ottawa, ON, Canada). H9C2 cells were seeded in six-well plates and allowed to adhere for $24 \mathrm{~h}$. Cells (50\% confluent) were transfected with a mixture containing Lipofectamine 2000 (Life Technology), OPTIMEM (Life Technology) and siRNA. The siRNA/Lipofectamine complex was then added to the medium. The final concentration of siRNA was $10 \mathrm{mM}$. Cells were incubated for $4-6 \mathrm{~h}$ at $37^{\circ} \mathrm{C}$ and fresh medium was then added.

\section{Autophagy detection}

Autophagy flux was analyzed by western blot analysis cells with antibodies against Light chain 3B (LC3B, 1:1,000) and p62 $(1: 1,000)$ antibodies were purchased from Novus Biologicals (Centennial, USA). Details see "Western blot assay".

In addition, the autophagic puncta accumulation in the cytoplasm was detected using the Autophagy Detection kit in accordance with the manufacturer's instructions (Abcam, USA). Briefly, cells were maintained in serum-free Earle's Balanced Salt Solution for 2 hour to induce autophagy. Then cells were fixed using $4 \%$ paraformaldehyde fixative. The autophagy status was evaluated Briefly, cells were grown to $70 \%$ confluence and were stained with Autophagy Detection Reagent and Hoechst 33342 nuclear dye solution at $37^{\circ} \mathrm{C}$ for $30 \mathrm{~min}$ in the dark before being imaged with a Nikon TE2000 Microscope (Nikon, Tokyo, Japan). Slides were sealed with coverslip using mounting medium and then analyzed by confocal microscopy. Confocal images were collected on Nikon A1 microscope using a 60x oil immersion objective lens and NIS Elements software.The total numbers of puncta in the cytoplasm were quantified using Image $\mathrm{J}$ and normalized by cell number in each field. A total of 5 fields were measured for each cell group and assays were repeated 3 times.

\section{Intracellular Reactive oxygen species (ROS) determination}

To detect intracellular ROS, cells $(5 \times 104$ cells $/ \mathrm{ml}, 100 \mu \mathrm{l} /$ well $)$ were seeded in a 96 -well black plate. After treatment, cells were incubated with $10 \mu \mathrm{M} \mathrm{DCFH}-\mathrm{DA}$ probe at $37^{\circ} \mathrm{C}$ for $20 \mathrm{~min}$. Then cells were washed twice with PBS. The fluorescence was read with a fluorescence microplate reader at excitation/emission of $535 / 610 \mathrm{~nm}$.

\section{Co-Immunoprecipitations analyses}


For co-immunoprecipitation assays, cells were lysed by sonication. A total of 100 ug Protein G SureBeads Magnetic Beads suspensions (BIO-RAD, Hercules, California) were incubated while rolling with 5 ug mouse monoclonal anti-Beclin 1 and rabbit monoclonal anti-FAK antibody (AbCam, USA) for 2 hours at room temperature. The beads were precipitated on a magnetic rack and washed three times with PBST solution (PBS $+0.1 \%$ Tween 20). The lysates then were added into the magnetic beads for $1 \mathrm{~h}$ rolling incubation at room temperature. The beads were precipitated again and washed three times with PBST solution. The immunoprecipitates were separated from the beads by using Glycine elution buffer $(20 \mathrm{mM}$, PH 2.0), followed by $10 \%$ SDS Polyacrylamide Gel Electrophoresis (SDS-PAGE) and transferred onto polyvinylidene difluoride nylon membranes. The immunoprecipitates were probed with anti-mouse and anti-rabbit IgG, respectively (Santa Cruz, CA, USA). Protein bands were detected using an enhanced chemiluminescence detection kit (Pierce, Rockford, IL, USA).

\section{Statistical Analysis}

An analysis of variance (ANOVA) was used on all variables to determine whether significant differences existed between groups. When a significant F-ratio was obtained, a Tukey HSD post hoc test was used to identify statistically significant differences $(p<0.05)$ among the means. Statistical analyses were performed using JMP software (SAS Institute Inc., Cary, NC), and all values are expressed as means \pm SE.

\section{Results}

OPG induced cellular hypertrophy

In consistence with previous studies, we found that OPG treatment at an increasing concentration stimulates cells to a hypertrophic phenotype, indicated by increased cellular surface and protein content per cell in a dose dependent manner. The pro-hypertrophic effect started at the concentration of $25 \mathrm{ng} / \mathrm{mL}$ and reaches maximum effect at $250 \mathrm{ng} / \mathrm{mL}$ for both cellular surface and protein content per cell (Fig. 1A and 1B).We then used $100 \mathrm{mg} / \mathrm{mL}$ OPG to treat cells at different time points. We found that OPG also includes hypertrophic response in a time-dependent manner, starting from 12 hours and reached maximum effect at $48 \mathrm{~h}$ thereafter and remains stable until 72 hours. In the following studies, we used $100 \mathrm{ng} / \mathrm{mL}$ for 24 hours as a treatment condition (Fig. 1C and 1D).

OPG inhibited autophagy

To investigate the effect of OPG on autophagy, we first checked the autophagic puncta accumulations in cells after OPG treatment. As shown in Fig. 2A, OPG treatment significantly attenuated the accumulation of autophagic puncta in $\mathrm{H} 9 \mathrm{C} 2$ cells in comparison to control cells without OPG treatment, suggesting 
OPG may exert a inhibitory effect on autophagy. To confirm this hypothesis, we next used the western blot analysis to determine changes in LC3-II and p62 in cultured H9C2 cells. LC3-II expression is a marker for complete autophagosomes, while p62 is a ubiquitin-binding protein involved in lysosome- or proteasome-dependent protein degradation. Treatment with OPG resulted in a considerable reduction in LC3-II, but increased p62 levels (Fig. 2B), suggesting OPG inhibits early stage of autophagosome formation. To confirm this finding, we used Baflomycin $A 1$ (Baf A1, $10 \mathrm{nM}$ for 6 hours ), a lysosome inhibitor, to conduct an autophagy flux assay. As shown in Fig. 2B, compared to cells treated with Baf A1 alone, BafA1 and OPG co-treatment did not increase the LC3-II expression and nor did it alter p62 levels, implying that OPG did not block the late stage LC3-II degradation, but hinders the early stage autophagosome formation. Taken together, the above data provide clear evidence to support the notion that OPG inhibits autophagy in cultured $\mathrm{H} 9 \mathrm{C} 2$ cells.

OPG induced hypertrophy is autophagy dependent

Increasing evidence has revealed an important pathogenic role of altered autophagy in cardiac hypertrophy[14, 16] and recent reports have overwhelmingly supported that autophagy insufficiency contributes to maladaptive cardiac remodeling and hypertrophy[21].

In order to clarify the role of autophagy in OPG-induced hypertrophy, we used 3-MA, an autophagy inhibitor and Rapamycin, an autophagy inducer, to see their effect on OPG induced hypertrophy. Interestingly, we found that Rapamycin attenuated the OPG-induced hypertrophy while 3-MA further enhanced this response, confirming that autophagy mediates OPG induced hypertrophy in $\mathrm{H} 9 \mathrm{C} 2$ cells.

OPG inhibited autophagy is not through ROS

We found that OPG increased the ROS level in cultured cells, indicated by DHE staining. ROS can inhibit autophagy by downregulating ULK1 mediated p53 pathway in selenite-treated NB4 cells[7]. Many chemical compound modulate autophagy through ROS pathway $[4,10,13,24]$. Thus, we test if ROS scavenger $\mathrm{N}$-acetylcysteine has any role in OPG inhibited autophagy. We did not see the co-treatment with $\mathrm{N}$-acetylcysteine justly slightly affects the autophagy status in cells treated with OPG, suggesting that OPG inhibited autophagy is not through ROS pathway (Data no shown).

OPG inhibited autophagy via FAK phosphorylation

Previous studies revealed that OPG activates the integrin/FAK signaling, resulting in the activation of Akt. Inhibition of both FAK and Akt signaling significantly inhibited OPG-mediated attenuation of TRAILinduced apoptosis[12]. OPG also stimulated ERK1/2 and p38 MAPK signaling pathways in different cell types[1, 6, 11, 23]. In cultured H9C2 cells, we found that treatment with OPG induced a robust increase in phosphorylation of FAK at Tyr-397 and Tyr-925, together with the increased PI3K, Akt, ERK and p38MAPK (Fig. 4A).

Subsequently, we silenced these above-mentioned proteins using si-RNA technique (Fig. 4B), to check their role in OPG induced autophagy. Of note, we found that siRNA-mediated knockdown of FAK fully 
abrogated the OPG-induced changes in p62 levels and LC3 modification as well as the puncta numbers in the cytoplasm (Fig. 4C and 4D). On the other hand, the knockdown of PI3K, Akt, ERK and MAPK did not, or slightly, if any, affect the effect of OPG on autophagy status and cell hypertrophy markers (data not shown).

OPG boosted FAK and Beclin1 interaction and prompts Beclin1 phosphorylate at 233

Beclin 1 is a Core Protein regulating autophagy. It has been demonstrated that phosphorylation of Beclin1 at Tyr233 limit its binding with the complex I and complex II, a necessary step for autophagy activation [5]. As shown in Fig. 5A, OPG treatment led to phosphorylation of Beclin1-Tyr-233 in cells (Fig. 5A,left). A recent study has identified the FAK binds Beclin1 and induces phosphorylation at Beclin-Tyr-233 site, we thus checked if FAK mediated OPG-induced phosphorylation of Beclin1-Tyr-233. As expected, with FAK silencing, OPG per se failed to phosphorylate Beclin1-Tyr-233 (Fig. 5A, right). Silencing Akt, activation of p38 mitogen-activated protein kinase (MAPK) and Phosphoinositide 3-kinases (PI3Ks) did changed the OPG induced phosphorylation of Beclin1-Tyr-233 and subsequent autophagy inhibition (data not shown). Next, we used Beclin1 mutant (Tyr-233 to Phe-233) to test the significance of Beclin1-Tyr-233 in OPG induced cellular hypertrophy. Compared to wild type controls, we observed that mutation of Tyr-233 to Phe-233 completely abolished OPG induced cellular hypertrophy, suggesting phosphorylation of Beclin1Tyr-233 is critical for OPG/FAK pathway in inducing hypertrophic response (Fig. 5B). To check if OPG affects the binding between FAK and Beclin1, we conducted the CO-IP assay. As shown in Fig. 5C, OPG enhanced the binding between FAK and Beclin1(Fig. 5C).

\section{Discussion}

Our previous study reported that OPG treatment stimulates cardiomyocyte hypertrophy but the mechanism remains large unknown. In this study, we found that FAK mediated OPG induced cardiomyocyte hypertrophy via suppression of Beclin 1 dependent autophagy. To the best of our knowledge, this is the first study reveal the significance of FAK and autophagy pathway in OPG induced cardiomyocyte hypertrophy.

OPG has been reported to attenuate TRAIL-induced apoptosis in a variety of cancer cells, including ovarian cancer cells. OPG-mediated protection against TRAIL has been attributed to its decoy receptor function. However, a recent study reveals that OPG can attenuate TRAIL-induced apoptosis in a TRAIL binding-independent manner, but through the activation of integrin/FAK/Akt signaling in OC cells. In pulmonary arterial hypertension animal model, OPG facilitates PAH pathogenesis by regulating pulmonary arterial smooth muscle cell proliferation, through Integrin av $33 / F A K / A K T$ Signaling. OPG induces cytoskeletal reorganization and activates FAK, Src, and ERK signaling in endothelial cells. Consistent with this finding, we observed that OPG induced FAK phosphorylation. Importantly, silencing FAK abolished the OPG induced autophagy suppression and hypertrophy response, suggesting FAK is the main mediator of OPG in this process. 
So far, how OPG affects autophagy in cardiomyocyte remains unclear. Previous studies revealed that OPG inhibits osteoclast differentiation and bone resorption by enhancing autophagy via AMPK/mTOR/p70S6K signaling pathway in vitro[19]. Also, OPG inhibits osteoclast bone resorption by inducing autophagy via the AKT/mTOR/ULK1 signaling pathway. Contrary to these studies, we found that OPG actually exerts an inhibitory effect on autophagy in $\mathrm{H} 9 \mathrm{C} 2$ cells. We postulate there may exist a cell type specific effect of OPG. Increasing evidence has revealed an important pathogenic role of autophagy in cardiac hypertrophy and heart failure. Although an early study suggested that cardiac autophagy is increased and that this increase is maladaptive to the heart subject to pressure overload, more recent reports have overwhelmingly supported that autophagy insufficiency contributes to cardiac remodeling and heart failure. Modulation autophagy has emerged as a new approach for the prevention and treatment of cardiac hypertrophy. For example, Oridonin protects against cardiac hypertrophy by promoting p21-related autophagy[22]. Hexarelin protects cardiac H9C2 cells from angiotensin Il-induced hypertrophy via the regulation of autophagy. Resveratrol prevents chronic intermittent hypoxia-induced cardiac hypertrophy by targeting the PI3K/AKT/mTOR pathway. In our study, we found that inhibition of autophagy by 3-MA dramatically further enhanced the OPG induced hypertrophic markers while Rapamycin rescued these changes, suggesting the importance of autophagy in OPG induced hypertrophic characteristics in $\mathrm{H} 9 \mathrm{C} 2$ cells.

In order to further elucidate the molecular mechanism OPG regulates autophagy and hypertrophy, we studied the interaction between FAK and Beclin1, a major autophagy regulator which plays a critical role in both autophagosome formation and autophagosome-lysosome fusion, and recent studies reveal that Beclin 1 serves as a nexus for autophagy regulation in response to various signaling pathways. ET $\mathrm{C}$. has identified Tyr-233 (a site highly conserved from lower vertebrates to mammals; Table 1) as the only FAK phosphorylation site on Belcin1. Indeed, in this study, we observed that FAK mediated the OPG induced Belcin1-Tyr-233 phosphorylation. It has been reported that phosphorylation of Beclin1 at Tyr-233 can restrain its binding ability to complex I (Atg14L/Vps15/Vps34) as well as complex II (UVRAG/Vps15/Vps34), which are essential steps for autophagosome formation and autophagosomelysosomal fusion, respectively [5]. So it is plausible to reason that OPG activates FAK, which in turn phosphorylates Belcin1-Tyr-233, causing inability to form complex I and II, thus hinders autophagy activation.

\section{Conclusions}

In summary, we discovered the mechanism under which OPG induces hypertrophic response in cultured H9C2 cells. Autophagy suppression via FAK/Beclin1 pathway may serve as target for the future therapeutic approaches against cardiac hypertrophy.

\section{Declarations}

\section{Ethics approval and consent to participate}


Not applicable.

\section{Availability of supporting data}

Not applicable.

\section{Competing interests}

None.

\section{Funding}

The study was supported by the Natural Science Foundation of Guangdong Province (No. 2018030310288) and the Scientific research start-up plan of Southern Medical University (No. QD2017N028).

\section{Authors' contributions}

AS and DZ conceived this project. AS,DZ and TL conducted the cellular study. AS and TZ analyzed the data and drafted this manuscript. All authors read and approved the final manuscript.

\section{Consent for publication}

All authors consent for publication.

\section{Acknowledgements}

We thank Dr Xuwei Hou at the University of Missouri for his help in statistics.

\section{Authors' information}

Not applicable.

\section{References}

1.

Arayatrakoollikit $\mathrm{U}$, Pavasant $\mathrm{P}$, Yongchaitrakul T. Thrombin induces osteoprotegerin synthesis via phosphatidylinositol 3'-kinase/mammalian target of rapamycin pathway in human periodontal ligament cells. J Periodontal Res. 2008;43:537-43.

doi:10.1111/j.1600-0765.2007.01071.x.

2.

Bezerra MC, Carvalho JF, Prokopowitsch AS, Pereira RM. RANK, RANKL and osteoprotegerin in arthritic bone loss. Braz J Med Biol Res. 2005;38:161-70. 
3.

Boyce BF, Xing L. Biology of RANK, RANKL, and osteoprotegerin. Arthritis Res Ther. 2007;9(Suppl 1):1. doi:10.1186/ar2165.

4.

Chen X, Hu Y, Zhang W, Chen K, Hu J, Li X, Liang L, Cai X, Hu J, Wang K, Huang A, Tang N. Cisplatin induces autophagy to enhance hepatitis $B$ virus replication via activation of ROS/JNK and inhibition of the Akt/mTOR pathway. Free Radic Biol Med. 2019;131:225-36.

doi:10.1016/j.freeradbiomed.2018.12.008.

5 .

Cheng Z, Zhu Q, Dee R, Opheim Z, Mack CP, Cyr DM, Taylor JM. Focal Adhesion Kinase-mediated Phosphorylation of Beclin1 Protein Suppresses Cardiomyocyte Autophagy and Initiates Hypertrophic Growth. J Biol Chem. 2017;292:2065-79.

doi:10.1074/jbc.M116.758268.

6.

Chung ST, Geerts D, Roseman K, Renaud A, Connelly L. Osteoprotegerin mediates tumor-promoting effects of Interleukin-1 beta in breast cancer cells. Mol Cancer. 2017;16:27. doi:10.1186/s12943-017-0606y.

7.

Ci Y, Shi K, An J, Yang Y, Hui K, Wu P, Shi L, Xu C. ROS inhibit autophagy by downregulating ULK1 mediated by the phosphorylation of p53 in selenite-treated NB4 cells. Cell Death Dis. 2014;5:e1542. doi:10.1038/cddis.2014.506.

8.

Erkol A, Pala S, Kirma C, Oduncu V, Dundar C, Izgi A, Tigen K, Gibson CM. Relation of circulating osteoprotegerin levels on admission to microvascular obstruction after primary percutaneous coronary intervention. Am J Cardiol. 2011;107:857-62. doi:10.1016/j.amjcard.2010.10.071.

9 .

Grajewski KG, Stojanovska J, Ibrahim EH, Sayyouh M, Attili A. Left Ventricular Hypertrophy: Evaluation With Cardiac MRI. Curr Probl Diagn Radiol. 2019. doi:10.1067/j.cpradiol.2019.09.005.

10.

Huang L, Yu LJ, Zhang X, Fan B, Wang FZ, Dai YS, Qi H, Zhou Y, Xie LJ, Xiao S. Autophagy regulates glucose-mediated root meristem activity by modulating ROS production in Arabidopsis. Autophagy. 2019;15:407-22.

doi:10.1080/15548627.2018.1520547.

11.

Jia D, Zhu Q, Liu H, Zuo C, He Y, Chen G, Lu A. (2017) Osteoprotegerin Disruption Attenuates HySuInduced Pulmonary Hypertension Through Integrin alphavbeta3/FAK/AKT Pathway Suppression. Circ Cardiovasc Genet 10.

doi:10.1161/CIRCGENETICS.116.001591.

12. 
Kobayashi-Sakamoto M, Isogai E, Holen I. Osteoprotegerin induces cytoskeletal reorganization and activates FAK, Src, and ERK signaling in endothelial cells. Eur J Haematol. 2010;85:26-35. doi:10.1111/j.1600-0609.2010.01446.x.

13.

Li Q, Yin Y, Zheng Y, Chen F, Jin P. Inhibition of autophagy promoted high glucose/ROS-mediated apoptosis in ADSCs. Stem Cell Res Ther. 2018;9:289.

doi:10.1186/s13287-018-1029-4.

14.

Li Z, Wang J, Yang X. Functions of autophagy in pathological cardiac hypertrophy. Int J Biol Sci. 2015;11:672-8.

doi:10.7150/ijbs. 11883 .

15.

Lu S, Weiser-Evans MCM. Nuclear Focal Adhesion Kinase. Circ Res. 2019;125:167-9. doi:10.1161/CIRCRESAHA.119.315395.

16.

Nishida K, Otsu K. Autophagy during cardiac remodeling. J Mol Cell Cardiol. 2016;95:11-8. doi:10.1016/j.yjmcc.2015.12.003.

17.

Richey PA, Brown SP. Pathological versus physiological left ventricular hypertrophy: a review. J Sports Sci. 1998;16:129-41.

doi:10.1080/026404198366849.

18.

Shen A, Hou X, Yang D, Liu T, Zheng D, Deng L, Zhou T. Role of osteoprotegerin and its gene polymorphisms in the occurrence of left ventricular hypertrophy in essential hypertensive patients. Medicine. 2014;93:e154.

doi:10.1097/MD.0000000000000154.

19.

Tong X, Gu J, Song R, Wang D, Sun Z, Sui C, Zhang C, Liu X, Bian J, Liu Z. Osteoprotegerin inhibit osteoclast differentiation and bone resorption by enhancing autophagy via AMPK/mTOR/p70S6K signaling pathway in vitro. J Cell Biochem. 2018. doi:10.1002/jcb.27468.

20.

Wang W, Lollis EM, Bordeleau F, Reinhart-King CA. Matrix stiffness regulates vascular integrity through focal adhesion kinase activity. FASEB J. 2019;33:1199-208. doi:10.1096/fj.201800841R. 21.

Wang X, Cui T. Autophagy modulation: a potential therapeutic approach in cardiac hypertrophy. Am J Physiol Heart Circ Physiol. 2017;313:H304-19. doi:10.1152/ajpheart.00145.2017.

22.

Xu M, Wan CX, Huang SH, Wang HB, Fan D, Wu HM, Wu QQ, Ma ZG, Deng W, Tang QZ. (2019) Oridonin protects against cardiac hypertrophy by promoting P21-related autophagy. Cell Death Dis 10. doi:ARTN 40310.1038/s41419-019-1617-y. 
23.

Zhao H, Liu X, Zou H, Dai N, Yao L, Gao Q, Liu W, Gu J, Yuan Y, Bian J, Liu Z. Osteoprotegerin induces podosome disassembly in osteoclasts through calcium, ERK, and p38 MAPK signaling pathways. Cytokine. 2015;71:199-206. doi:10.1016/j.cyto.2014.10.007.

24.

Zhou Y, Wang Y, Zhou W, Chen T, Wu Q, Chutturghoon VK, Lin B, Geng L, Yang Z, Zhou L, Zheng S. YAP promotes multi-drug resistance and inhibits autophagy-related cell death in hepatocellular carcinoma via the RAC1-ROS-mTOR pathway. Cancer Cell Int. 2019;19:179. doi:10.1186/s12935-019-0898-7.

\section{Abbreviations}

Osteoprotegerin, OPG

Focal adhesion kinase ,FAK

extracellular matrix ,ECM

Tumor necrosis factor-related apoptosis-inducing ligand,TRAIL

Reactive oxygen species, ROS

steroid receptor coactivator, Src

extracellular signal-regulated kinases, ERKs

receptor activator for nuclear factor-KB ligand, RANKL

activation of p38 mitogen-activated protein kinase ,MAPK

Phosphoinositide 3-kinases,PI3Ks

Baflomycin A1, Baf A1

\section{Figures}




\section{Figure 1}

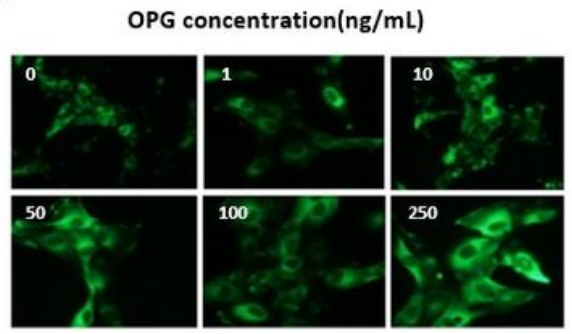

C

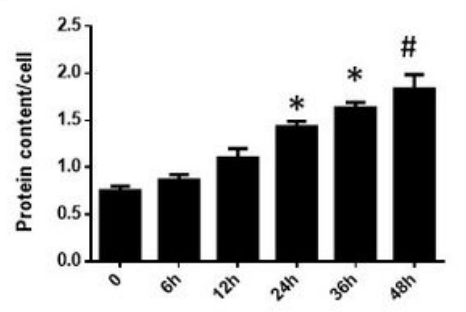

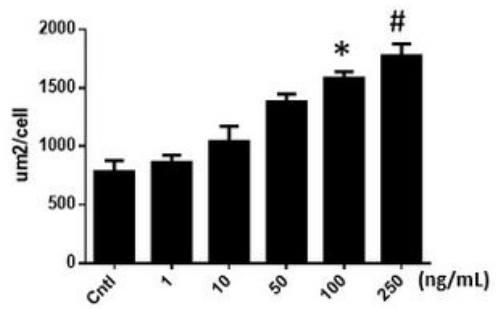

D

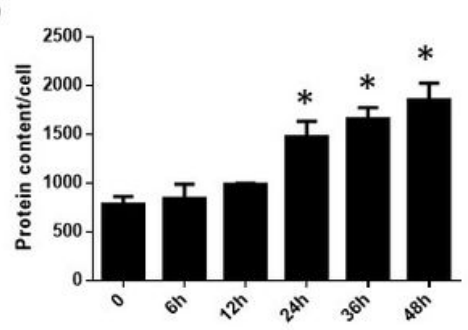

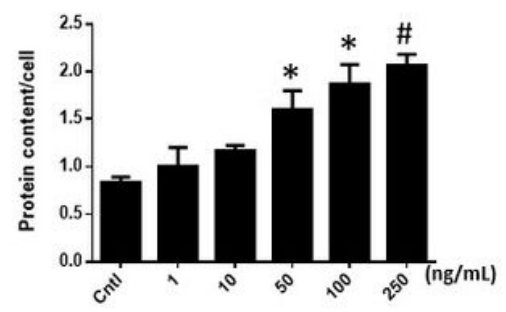

C

\section{Figure 2}

OPG stimulates hypertrophy in cultured $\mathrm{H} 9 \mathrm{C} 2$ cells in a dose and time dependent manner. A, Typical images for cell hypertrophy with OPG treatment at different concentrations. Cells were stained with fluorescein isothiocyanate-conjugated Phalloidin for 30 min.. *, $p<0.05$; $* *, p<0.01$. NS, not significant. Data are mean \pm S.E. of three independent experiments. All data were analyzed using two-tailed Student's $t$ tests. B. The quantification of cellular size in cells subject to OPG treatment with indicated concentrations. C. The quantification of protein content per cell in cells subject to OPG treatment with indicated concentrations. D. The quantification of cellular size in cells subject to OPG treatment $(100 \mathrm{ng} / \mathrm{mL})$ with indicated time points. E.The quantification of protein content per cell in cells subject to OPG treatment $(100 \mathrm{ng} / \mathrm{mL})$ with indicated time points. *, $p<0.05 ; \#, p<0.01$. Data are mean \pm S.E. of three independent experiments.

A
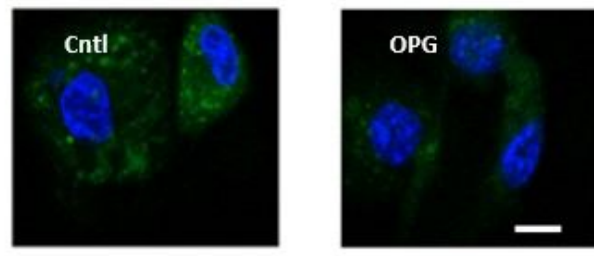

\section{Figure 2}

OPG inhibits autophagy in cultured $\mathrm{H} 9 \mathrm{C} 2$ cells.

A, Typical images for autophagic puncta accumulations (green florescence signals) in cells after OPG treatment. Cell nucleus were stained with DAPI (scale bar, 10uM).

B. The autophagy flux assay with application of lysosome inhibitor Baflomycin A1 (Baf A1) . 
Figure 4

OPG inhibits autophagy in cultured $\mathrm{H} 9 \mathrm{C} 2$ cells. A, Typical images for autophagic puncta accumulations (green florescence signals) in cells after OPG treatment. Cell nucleus were stained with DAPI (scale bar, 10uM). B. The autophagy flux assay with application of lysosome inhibitor Baflomycin A1 (Baf A1) .

A

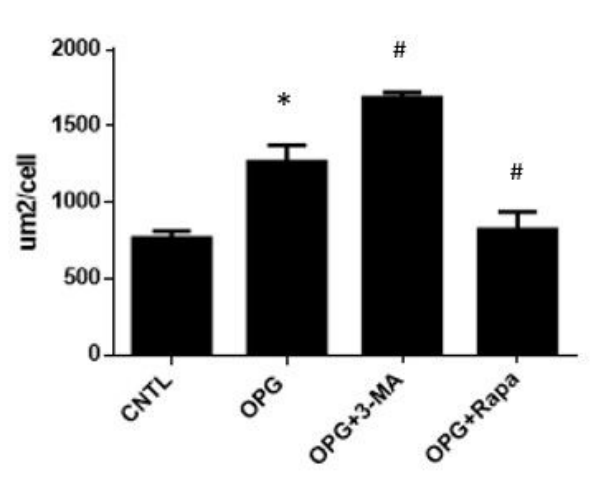

B

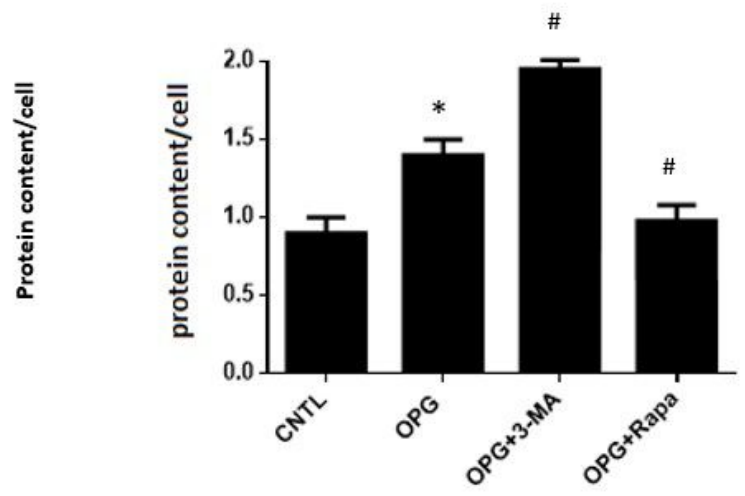

Figure 3

OPG inhibited autophagy contributes to hypertrophic responses in cultured $\mathrm{H} 9 \mathrm{C2}$ cells.

A. The quantification of cell size in cells subject to OPG; OPG+3-MA, an autophagy inducer; OPG+Rapa, an autophagy inhibitor.

B. The quantification of protein content per cell in cells subject to OPG; OPG+3-MA, an autophagy inducer;OPG+Rapa, an autophagy inhibitor.

*, $p<0.05$; vs CNTL, \#, $p<0.05$ vs OPG. Data are mean \pm S.E. of three independent experiments.

\section{Figure 6}

OPG inhibited autophagy contributes to hypertrophic responses in cultured $\mathrm{H} 9 \mathrm{C} 2$ cells. The quantification of cell size in cells subject to OPG; OPG+3-MA, an autophagy inducer; OPG+Rapa, an autophagy inhibitor. The quantification of protein content per cell in cells subject to OPG; OPG+3-MA, an autophagy inducer;OPG+Rapa, an autophagy inhibitor. ${ }^{*}, p<0.05$; vs CNTL, $\#, p<0.05$ vs OPG. Data are mean \pm S.E. of three independent experiments. 


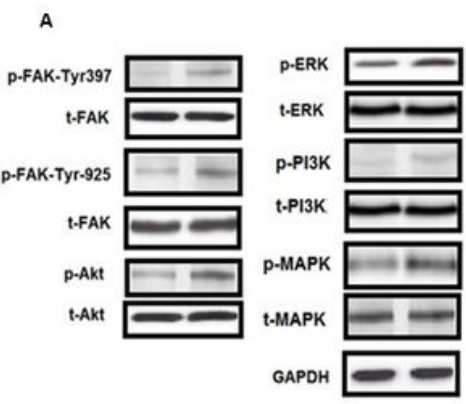

C

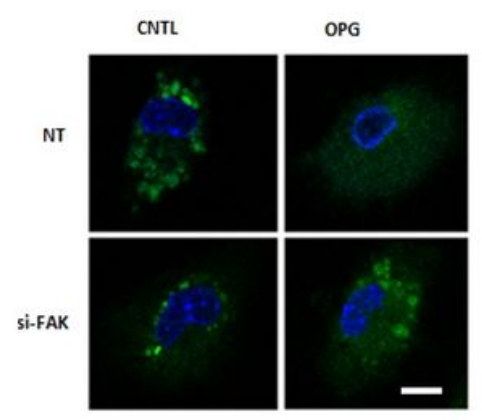

B

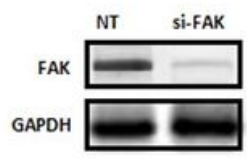

D

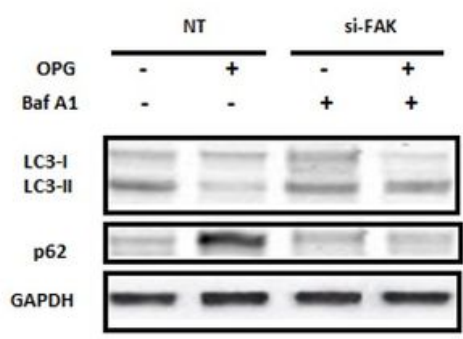

Figure 4.OPG inhibited autophagy via FAK

A. OPG induces phosphorylation of

FAK,Akt,ERK,PI3K and MAPK. Cell lysates

were subject to Western blot assay with

proper phosphorylated and total antibodies.

B. Silencing FAK using si-RNA technology. NT,

non target RNA sequence.

C. Autophagic puncta in cells using confocal

image technique. Scale bar $=20$ um.

D. Autophagy flux assay using western blot

assay. $C$ and $D$ showed that $\mathrm{OPG}$ alone

induced p62 accumulation and LC3-II

reduction were abrogated in cells with si-FAK

transfection.

\section{Figure 8}

OPG inhibited autophagy via FAK A. OPG induces phosphorylation of FAK,Akt,ERK,PI3K and MAPK. Cell lysates were subject to Western blot assay with proper phosphorylated and total antibodies. B. Silencing FAK using si-RNA technology. NT, non target RNA sequence. C. Autophagic puncta in cells using confocal image technique. Scale bar =20um. D. Autophagy flux assay using western blot assay. $C$ and $D$ showed that OPG alone induced p62 accumulation and LC3-II reduction were abrogated in cells with si-FAK transfection. 
A

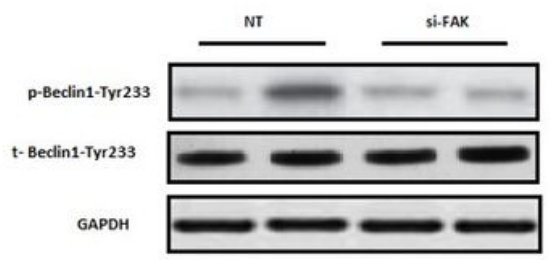

Figure 5. OPG boosts the FAK and Beclin1 interaction and prompts Beclin1 phosphorylate at 233.

A. OPG treatment led to phosphorylation of Beclin1-Tyr-233, while FAK silencing abrogates this effect.

B. mutation of Beclin1 Tyr-233 to Phe-233 completely abolished OPG induced cellular hypertrophy, indicated by cellular surface and protein content per cell.

c. Co-Immunoprecipitation assay show that OPG enhances the FAK and Beclin1 mutual binding.
B
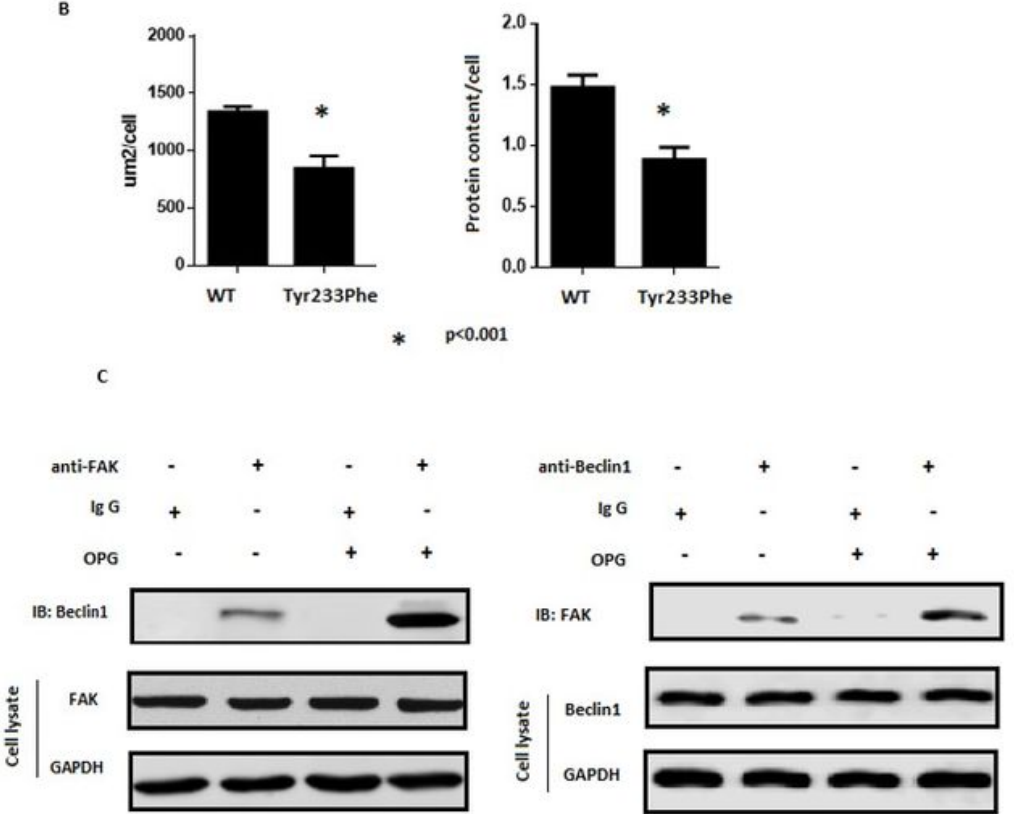

\begin{tabular}{l|l} 
: & Beclin1 \\
$\overline{\bar{z}}$ & \\
GAPDH
\end{tabular}

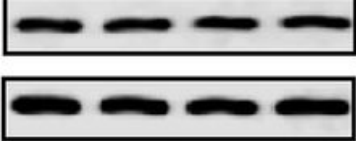

\section{Figure 10}

OPG boosts the FAK and Beclin1 interaction and prompts Beclin1 phosphorylate at 233. OPG treatment led to phosphorylation of Beclin1-Tyr-233, while FAK silencing abrogates this effect. mutation of Beclin1 Tyr-233 to Phe-233 completely abolished OPG induced cellular hypertrophy, indicated by cellular surface and protein content per cell. Co-Immunoprecipitation assay show that OPG enhances the FAK and Beclin1 mutual binding. 